\title{
Concerning long-term geomagnetic variations and space climatology
}

\author{
K.-H. Glassmeier ${ }^{1,2}$, J. Vogt $^{2}$, A. Stadelmann ${ }^{3}$, and S. Buchert ${ }^{4}$ \\ ${ }^{1}$ Institut für Geophysik und extraterrestrische Physik, TU Braunschweig, Germany \\ ${ }^{2}$ Max-Planck-Institut für Aeronomie, Katlenburg-Lindau, Germany \\ ${ }^{3}$ School of Engineering and Sciences, International University of Bremen, Germany \\ ${ }^{4}$ Swedish Space Research Institute, Uppsala, Sweden
}

Received: 14 May 2003 - Revised: 2 July 2004 - Accepted: 15 August 2004 - Published: 3 November 2004

\begin{abstract}
During geomagnetic polarity transitions the surface magnetic field of the Earth decays to about $25 \%$ and less of its present value. This implies a shrinking of the terrestrial magnetosphere and posses the question of whether magnetospheric magnetic field variations scale in the same manner. Furthermore, the geomagnetic main field also controls the magnetospheric magnetic field and space weather conditions. Long-term geomagnetic variations are thus intimately related to space climate. We critically assess existing scaling relations and derive new ones for various magnetospheric parameters. For example, we find that ring current perturbations do not increase with decreasing dipole moment. And we derive a scaling relation for the polar electrojet contribution, indicating a weak increase with increasing internal field. From this we infer that the ratio between external and internal field contributions may be weakly enhanced during polarity transitions. Our scaling relations also provide more insight on the importance of the internal geomagnetic field contribution for space climate.
\end{abstract}

Key words. Geomagnetism and paleomagnetism (paleomagnetic secular variation) - Magnetospheric physics (current systems; magnetospheric configuration and dynamics)

\section{Introduction and motivation}

Paleomagnetic studies found convincing evidence for geomagnetic polarity transitions, with the last one, the Matuyama-Brunhes transition, occurring about 780000 years ago (e.g. Merrill and McFadden, 1999). During such a polarity transition, the mean intensity of the dipole field decreases to at least $\sim 25 \%$ or less of its present value. Siscoe and Chen (1975) were the first to study, in more detail, the consequences of a polarity transition induced field decrease on the terrestrial magnetosphere. As the standoff distance $R_{M P}$ of the dayside magnetopause scales as

Correspondence to: K.-H. Glassmeier

(kh.glassmeier@tu-bs.de
$R_{M P} \propto M^{1 / 3}$, where $M$ is the magnetic dipole strength, Siscoe and Chen (1975) suggested that the magnetopause of such a paleomagnetosphere is located at about $8 R_{E}$ and even closer to the Earth. The present day value is $R_{M P} \approx 10 R_{E}$.

Space weather describes the conditions in space that affect Earth and its technological systems, for example, power lines and communication satellites (e.g. Pirjola, 2002). Space weather is a consequence of the behavior and dynamics of the Sun, the nature of the geomagnetic main field and the terrestrial magnetosphere and atmosphere. Long-term variations of the main field may thus be as important for space weather as long-term variations of solar activity, that is longterm variations of the geomagnetic field are of paramount importance for our understanding of space climate. Scaling relations for various processes and parameters of the magnetosphere are thus very important for our ability to analyse past and forecast future space weather conditions and to evaluate whether the internal geomagnetic field contribution or solar forcing is more important for space climate considerations.

Besides a scaling relation for the magnetopause distance, other scaling relations for various magnetospheric parameters have already been suggested. Siscoe and Chen (1975) argue that the polar cap width scales with $\cos \vartheta \propto M^{-1 / 6}$, where $\vartheta$ is the latitude of the polar cap boundary. Vogt and Glassmeier (2001) derive a somewhat different scaling for the polar cap width by using the ansatz $R_{T} \propto M^{\gamma}$ to describe the dependence of the tail radius $R_{T}$ from M; here, $\gamma$ is a scaling exponent. Equating the magnetic fluxes through the polar cap and the tail gives one a scaling relation for the polar cap width: $\cos \vartheta \propto M^{\gamma-1 / 2}$. Siscoe and Chen's (1975) estimate implies $\gamma=1 / 3$. A corresponding treatment of the problem by Siebert (1977) gives one $\gamma=0$, that is the tail radius is found to be independent of the magnetic dipole moment. Based on the recent work by Roelof and Sibeck (1993), Vogt and Glassmeier (2001) suggest $\gamma=1 / 2$ for periods of strong southward interplanetary magnetic field (IMF).

Siscoe and Chen (1975) also tackle the question of ring current variations and estimate that the number and strength of magnetic storms increases with decreasing $M$. They estimate that the contribution of the storm-time ring current 


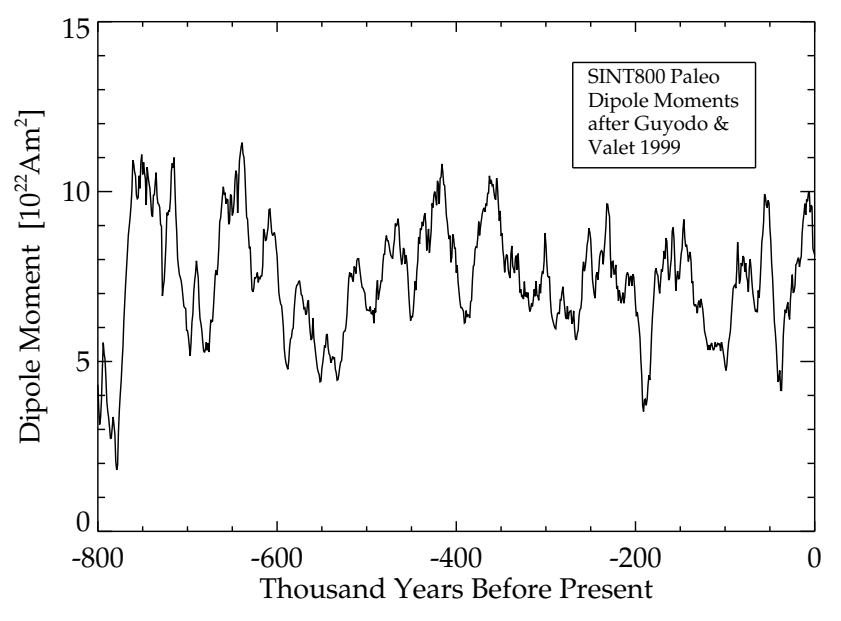

Fig. 1. Dipole moments of the geomagnetic field over the past 800000 years based on relative paleointensities of SINT800 (after Guyodo and Valet, 1999).

to the equatorial surface magnetic field may be as large as $42 \%$ of the equatorial geomagnetic field during a polarity transition.

Indeed, paleomagnetic studies reveal that occasionally brief episodes of extremely rapid field changes are recorded in paleomagnetic data during times of polarity transitions (Coe and Prevot, 1989; Coe et al., 1995). Changes of $300 \mathrm{nT}$ and several degrees per day in a paleomagnetic recording as the Steens Mountain reversal record is hard to be explained as a result of changes in the Earth's outer core and its dynamo, but most probably require external magnetic field variations as an explanation (Ultré-Guérard and Achache, 1995; Jackson, 1995).

Other current systems in the magnetosphere-ionosphere system, such as the polar and the equatorial electrojets, may as well contribute significantly to the total geomagnetic field at the Earth's surface. This raises the question of whether external geomagnetic variations may become as large as the internal magnetic field strength during polarity transitions. If this hypothesis holds, then major consequences not only for the interpretation of paleomagnetic data will result, but also for the shape and dynamics of the inner magnetosphere, now not mainly governed by the main field, but also by the selfexcited external field contributions.

Besides Siscoe and Chen's (1975) scaling relation for the ring current, no other scaling relations for current systems in the magnetosphere-ionosphere system have been derived. This motivates the present study in which we shall derive basic scaling relations for external geomagnetic variations due to polar and equatorial electrojet currents and their dependencies on the internal magnetic field strength, to explore in more detail the importance of long-term geomagnetic field variations for the space climate.

First, we shall derive a scaling law for the ring current contribution which differs from earlier treatments by Siscoe and Chen (1975) and Ultré-Guérard and Achache (1995).
Second, the ground-magnetic effect of the polar electrojets (PEJ) and its dependence of the magnetic dipole moment is discussed. Third, the equatorial electrojet contribution and the Sq-variations are analyzed. To all the various scaling laws we apply estimates of the dipole strength over the past 800000 years based on relative paleointensities of the SINT800 record (Guyodo and Valet, 1999; Fig. 1) and derive the long-term variation of various magnetospheric parameters. It should be noted that the SINT800 data set includes data from the last geomagnetic polarity transition, the Matuyama-Brunhes transition.

As an example Fig. 2 displays the magnetopause position using the Siscoe and Chen (1975) scaling relation. To account for variations of the magnetopause position due to solar wind dynamic pressure changes observations of long-term solar wind flow variations by Gazis (1996) are used. One hundred-day averages of the solar wind speed at various distances from the Sun indicate a variability of the mean flow speed of the order of $\pm 150 \mathrm{~km} / \mathrm{s}$. We used this value to give error bars in Fig. 2, indicating the solar wind driven variation of the magnetopause position.

However, under very extreme conditions the magnetopause can be observed even within the geostationary orbit. In Fig. 2 the position under the extreme conditions of 4 May 1998 is indicated (for details of this event, see Russell et al., 2000). It can be seen from Fig. 2, that even during a polarity transition such as the Matuyama-Brunhes transition, the magnetopause under average solar activity conditions never moves closer than about $5 \mathrm{R}_{E}$. During very strong solar wind activity the magnetopause can be observed at these positions even today. Space climate variations of the magnetopause distance, as caused by long-term geomagnetic field conditions, are thus within current, shorter term space weather variations. It is solar activity which has a more important influence on the magnetopause position.

This implies the question of whether this is true for all the magnetospheric parameters discussed. In particular, we are interested in a more detailed discussion of the strength of the external magnetic field contributions compared to the internal ones. We are thus concluding our contribution with a discussion of the possible effects of the various external geomagnetic field contributions.

In all the following discussions we shall assume that the terrestrial field is of a pure dipole nature. Non-dipolar contributions will be neglected for the time being. This results in a considerable simplification of the problem treated but allows for a much more straightforward derivation of firstorder scaling relations. It is our intention to study the possible significance of long-term geomagnetic field variations on major magnetospheric parameters. Long-term variations of solar activity might be equally or even more important. Also, the effect of energetic particles in the magnetosphere is not discussed here. A first discussion on this can be found in the work by Vogt and Glassmeier (2000). 


\section{The ring current contribution}

Siscoe and Chen (1975) pointed out that a scaling for the ring current magnetic field at the Earth's surface can be derived from the Dessler-Parker-Sckopke theorem (e.g. Sckopke, 1966; Wolf, 1995).

$D_{s t} \propto \frac{W_{R C}}{M}$.

Here, $D_{s t}$ is a measure for the ring current associated surface magnetic field and $W_{R C}$ is the total energy of the ring current particles. A priori it is not justified to assume that $W_{R C}$ is independent of $\mathrm{M}$, the magnetic dipole strength. Siscoe and Chen's (1975) scaling, $D_{s t} \propto M^{-1 / 3}$, implies $W_{R C} \propto M^{2 / 3}$, a result based on the assumption that the total ring current energy is proportional to the energy delivered to the magnetospheric system by the solar wind. This energy input scales with $R_{T}^{2}$, the tail cross section. Using $R_{T} \propto R_{M P}$ gives one Siscoe and Chen's (1975) scaling relation (see also UltréGuérard and Achache, 1995).

A different scaling results if one takes into account that not only the cross section of the magnetosphere, $R_{T}^{2}$, is decreasing with decreasing magnetic moment, but also the volume of that part of the magnetosphere where particle trapping is possible. If this volume is smaller, less particles can be trapped in the ring current region of the magnetosphere. Here, we assume that this volume, $V_{R C}$, scales as $V_{R C} \propto R_{M P}^{3} \propto M$. Following Vogt and Glassmeier (2001) we use $R_{T} \propto M^{\gamma}$, which gives one

$W_{R C} \propto R_{T}^{2} R_{M P}^{3} \propto M^{2 \gamma+1}$.

Here, $\gamma>0$. With these modifications the scaling relation for the ring current magnetic field is given by

$D_{s t} \propto M^{2 \gamma}$,

that is, in contrast to the work of Siscoe and Chen (1975) and Ultré-Guérard and Achache (1995) we find that the ring current associated magnetic field decreases with decreasing dipole magnetic moment.

Taking into account the volume of the ring current is also supported by considering the Burton equation $d D_{s t} / d t=Q(t)-D_{s t} / \tau$, where $Q(t)$ is a source function and $\tau$ denotes the decay rate due to charge-exchange loss of ringcurrent particles through collisions with neutral geocorona particles (Burton et al., 1975). The steady-state solution of this equation gives one $D_{s t}=Q \cdot \tau$. Now $\tau$ can depend on the dipole strength, since if the magnetosphere is smaller, the ring current forms closer to the Earth, where charge exchange occurs. Thus, $\tau$ should decrease with decreasing magnetospheric volume and dipole moment (G. Siscoe, personal communication). We also expect $Q$ to decrease with decreasing dipole strength. Thus, $D_{s t}$ significantly decreases with decreasing magnetic field.

The new scaling relation discussed here furthermore guarantees that for vanishing magnetic moment also, the ring current vanishes, a result most reasonable as the ring current requires particle trapping in a magnetic mirror topology. The

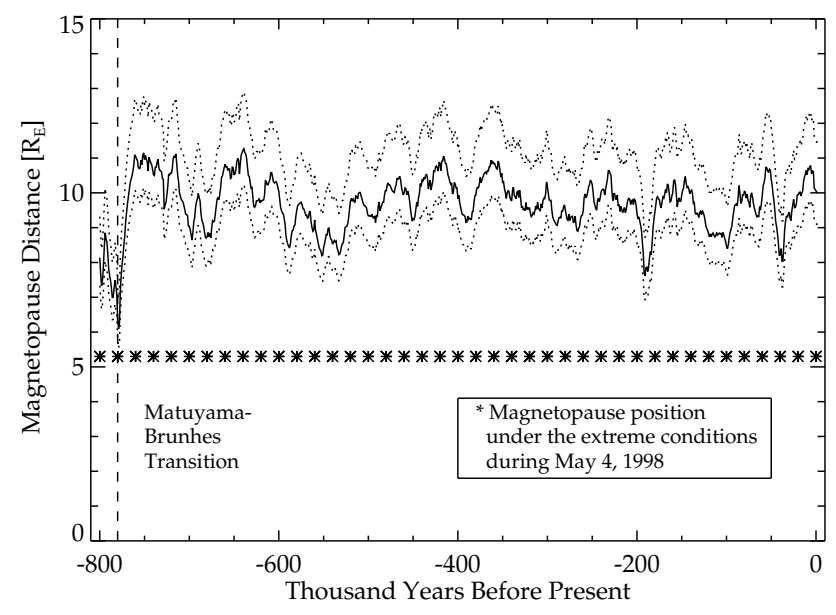

Fig. 2. Estimated magnetopause position during the past 800000 years using the Siscoe and Chen (1975) scaling relation. The error bars indicate the variability of the magnetopause position due to solar wind dynamic pressure variations and have been derived from long-term solar wind flow variations.

new scaling is also supported by observations in the Hermean magnetosphere, indicating that planet Mercury, with its much smaller internal magnetic field, does not support any large ring-current (Russell et al., 1988; Glassmeier, 2000). Ring current magnetic effects are thus of minor importance during polarity transitions following the approach discussed above.

\section{Scaling the convection electric field}

As we aim at scaling the strength of the PEJ magnetic field we need a proper scaling for the convection electric field in the ionosphere driving these current systems. The ionospheric electric field $\boldsymbol{E}_{\text {Iono }}$ is determined by the magnetospheric convection electric field $\boldsymbol{E}_{\boldsymbol{c}}$, which is related to the solar wind electric field $\boldsymbol{E}_{s w}=-\boldsymbol{v}_{s w} \times \boldsymbol{B}_{s w}$ via $E_{c}=\eta E_{s w}$, where $\eta \approx 0.2$ is the magnetic reconnection efficiency, and $\boldsymbol{v}_{s w}$ and $\boldsymbol{B}_{s w}$ denote the solar wind flow velocity and the interplanetary magnetic field, respectively (e.g. Siscoe and Chen, 1975). Here, we estimate the electric field via the potential drop $\Phi$ across the dayside magnetopause:

$\Phi \propto v_{s w} \cdot B_{s w} \cdot R_{M P}$.

We use the magnetopause distance $R_{M P} \propto M^{1 / 3}$ as a measure of the extension of the dayside magnetopause reconnection region. Solar wind velocity and interplanetary magnetic field magnitude are assumed as constant here. The ionospheric electric field in polar regions scales according to

$E_{\text {Iono }} \propto \Phi / 2 R_{E} \cos \vartheta \propto M^{5 / 6-\gamma}$,

where the divisor $2 R_{E} \cos \vartheta$ gives the polar cap width to which the potential is applied. For any value of $\gamma$ considered above, we conclude that the ionospheric electric field increases with increasing dipole moment. 
The simple scaling above does not at all take into account any effect of the ionospheric-magnetospheric current system generated. To first order the region 1 current system can be described as a figure- 8 meridional current system (Siscoe et al., 2002), whose magnetic field exhibits a southward field component at the magnetopause in the equatorial plane. Dayside reconnection is thus diminished due to these currents and saturation of the transpolar electric potential results. Siscoe et al. (2002) provide a suitable model on how to account for these effects. Based on what is called by them the Hill model (Hill et al., 1976), they derive an expression for the saturation of the transpolar potential scaling with $\mathrm{M}$ as

$\Phi_{s} \propto M^{4 / 3} / \Sigma_{0}$,

where $\Sigma_{0}$ is the constant of proportionality in a relation scaling the Pedersen conductance with $\mathrm{M}, \Sigma_{P}=\Sigma_{0} / M$. For details of the conductance scaling, see Sect. 4. From this a suitable scaling relation for the saturation ionospheric electric field is

$E_{\text {Iono }, \mathrm{s}} \propto \Phi_{s} / \cos \vartheta \propto \frac{1}{\Sigma_{0}} M^{-\gamma+11 / 6}$,

or, $E_{\mathrm{Iono}, \mathrm{s}} \propto M^{13 / 6}$, if $\Sigma_{0}$ is assumed independent from $\mathrm{M}$ and $\gamma=1 / 3$ is used. Thus, the saturation ionospheric electric field would decrease with decreasing dipole field.

Our derivation of a scaling for the ionospheric electric field and the magnetospheric convection electric field assumes that the magnetospheric convection pattern does not vary much during a polarity transition, an assumption valid only if the magnetospheric magnetic field is still dominated by a magnetic dipole aligned with the rotation axis. If quadrupole or octupole contributions from the internal field become important or even dominate the field or if the orientation of the dipole varies with respect to the Sun-Earth line, then the convection pattern may be much different. Thus, we are merely scaling the magnetic field strength dependence, but not its topology change. However, it should be noted that using the Hill model scaling and saturation conditions takes into account quadrupole field contributions resulting from the region 1 magnetosphere-ionosphere current system.

Scaling the convection electric field potential gives one a scaling relation for the plasmapause position. Following Siscoe and Chen (1975) we identify the plasmapause as the $\Omega$-pause (Brice, 1967), where the saturated convection potential $\Phi_{s} \propto M^{4 / 3} / \Sigma_{0}$ equals the potential of the corotational electric field $\Phi_{\text {cor }} \propto M / r$, where $\mathrm{r}$ denotes radial distance. With $\Phi_{s}=\Phi_{\text {cor }}$ the plasmapause position $R_{p p}$ scales as

$R_{p p} \propto M^{3 \gamma-11 / 6}$

where a scaling relation for $\Sigma_{0}$, as introduced in the next section has been used. With $\gamma=1 / 3$ the plasmapause position scales as

$R_{p p} \propto M^{-5 / 6}$

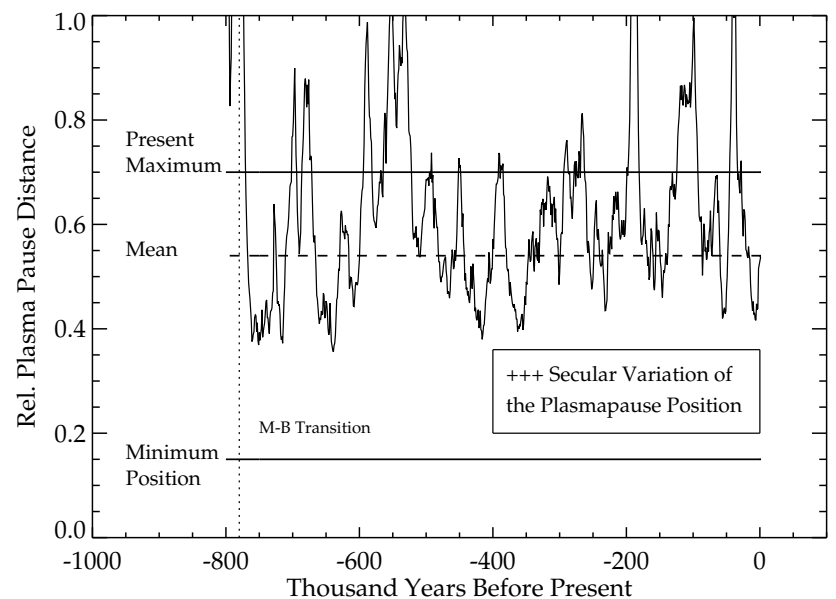

Fig. 3. Estimated relative plasmapause position over the past 800000 years using the Siscoe and Chen (1975) scaling relation, together with the current day plasmapause position and its variability.

that is, the plasmapause distance increases with decreasing M. This scaling is different from the previous scaling derived by Siscoe and Chen (1975), a result based on taking into account the saturation of the transpolar potential. In the original approach by Siscoe and Chen (1975) this effect was not considered and the convection potential was assumed independent from the internal geomagnetic field contribution.

Figures 3 displays the likely mean position, $r_{p p}$, of the plasmapause over the past 800000 years, normalized to the magnetopause position: $r_{p p}=\mathrm{R}_{p p} / R_{M P} \propto M^{-7 / 6}$, if $\gamma=1 / 3$ is assumed. The figure also indicates the variability of the current plasmapause position as observed by, for example, Laakso and Jarva (2001) and Moldwin et al. (2002). We conclude that relative variations introduced by secular changes in the dipole moment are much larger than changes introduced by magnetospheric activity. During field lows the plasmapause position may coincide with the magnetopause or is even located at a larger radial distance. This is, of course, unreasonable and indicates that the scaling derived is only applicable for a certain range of dipole moment values. Nevertheless, our scaling relation indicates that the magnetosphere for smaller dipole moments may be much more rotation dominated than at the present time. The paleomagnetosphere during a reversal is possibly a weak Jovian-type magnetosphere.

\section{The polar electrojets}

Current systems causing major geomagnetic field variations are the polar electrojets in the auroral zones. During disturbed times magnetic field variations of up to $2000 \mathrm{nT}$ have been observed (Allen et al., 1989). The sudden onset of field variations of this strength will cause pronounced geomagnetically induced current (GIC) effects (e.g. Pirjola, 2002). Such GIC effects depend on both the rise time of the perturbation, 
as well as the maximum strength of the inducing magnetic field. Our scaling relation for the polar electrojet strength allows, for example, one to estimate the maximum strength contribution to GICs.

On the average PEJ variations as estimated using the $\mathrm{AE}$ index are of the order of $200 \mathrm{nT}$. At auroral latitudes the internal field contribution is about $50000 \mathrm{nT}$, that is, the present ratio external to internal field contribution is of the order of $1 / 250$. Therefore, such external variations are usually neglected when interpreting paleomagnetic data.

Polar electrojet magnetic field variations are mainly determined by the ionospheric Hall currents (e.g. Glassmeier, 1987; Kamide and Baumjohann, 1993):

$b_{G} \propto \Sigma_{H} E_{\text {Iono }}$,

where $b_{G}$ is the magnitude of the horizontal component of the ground magnetic field, $E_{\text {Iono }}$ the ionospheric electric field strength, and $\Sigma_{H}$ the height-integrated Hall conductivity. For simplicity, $\Sigma_{H}$ is assumed to be uniform. Equation (10) demonstrates that a scaling law for $b_{G}$ requires scaling relations for both, the conductance $\Sigma_{H}$ and the electric field $E_{\text {Iono. }}$

It should be noted that when deriving Eq. (10) we made use of the so-called Fukushima-Boström-Vasyliunas theorem (Boström, 1964; Vasyliunas, 1970; Fukushima, 1976), stating that the ground magnetic effect is only due to the magnetic effect of the Hall currents, while the magnetic effect of the Pedersen currents is cancelled by the magnetic effects of the field-aligned and magnetospheric closure currents. This theorem strictly only holds for a uniform conductance distribution which, we think, is a fair approximation when deriving scaling relations.

The ionospheric Pedersen and Hall conductivities are significant in a layer where the ions are unmagnetized due to ion-neutral collisions and move partially along the electric field direction, and the electrons are magnetized performing nearly unperturbed $\boldsymbol{E} \times \boldsymbol{B}$ drifts. This difference in ion and electron motion gives rise to the Pedersen and Hall currents (Kelley, 1989, pp. 37). For magnetized electrons and partially unmagnetized ions the Pedersen and Hall conductivities are (Kelley, 1989, p. 39)

$\sigma_{P}=\frac{n_{e} e}{B} \frac{\kappa_{i}}{1+\kappa_{i}^{2}}$

and

$\sigma_{H}=\frac{n_{e} e}{B} \frac{\kappa_{i}^{2}}{1+\kappa_{i}^{2}}$,

where $n_{e}=n_{i}$ are the ion and electron number density, respectively, and $\kappa_{i, e}=\Omega_{i, e} / \nu_{i, e}$ are the ratios of the ion and electron gyro frequencies $\Omega_{i, e}$ to their collision frequencies $v_{i, e}$, respectively, and the approximation $\kappa_{e} \gg 1$ is made, which is valid at all heights above $75 \mathrm{~km}$ for the current geomagnetic field strength, and above about $85 \mathrm{~km}$ for a geomagnetic field reduced to $10 \%$ of its current strength. If the geomagnetic field becomes very low or even vanishes, then the collision frequencies dominate Eqs. (11) and (12) and the Hall conductivity becomes very small.

Equation (12) suggests a scaling relation

$\sigma_{H} \propto B^{-1} \propto M^{-1}$.

This scaling relation is justified if one assumes that $n_{e}(z)$ does not change very much with $B_{0}$, the background magnetic field. In the dayside ionosphere this assumption is certainly justified as $n_{e}(z)$ is mainly determined by solar insolation. In the nightside ionosphere the electron density depends very much on magnetospheric activity and particle precipitation.

The ground-magnetic field is determined by the heightintegrated conductivity and thus depends on the height distribution of the electron density and the collision frequencies. To receive a first estimate of the Pedersen and Hall conductance for the dayside ionosphere we assume an atmospheric and ionospheric structure as described in Kelley (1989) based on the following assumptions: Chapman layer with a maximum at $120 \mathrm{~km}$ and a scale height of $10 \mathrm{~km}$, isothermal barometric law for the neutral atmosphere density, normalized at $120 \mathrm{~km}$ according to the table in Kelley (1989, p. 461), temperature profile according to solar maximum conditions with a temperature of $176 \mathrm{~K}$ at a height of $90 \mathrm{~km}$, and collision frequencies for electron-neutral, and ion-neutral collisions as given in Appendix B of Kelley (1989).

For the standard ionospheric structure, the $\sigma_{P}$ layer does not change significantly its vertical shape, and its center moves up in altitude when the ionospheric magnetic field strength decreases. The vertical shape of the $\sigma_{H}$ layer also becomes thicker in addition to moving upwards. Therefore, when integrating numerically the $\sigma_{P}$ and $\sigma_{H}$ profiles over the height range $90-290 \mathrm{~km}$, we find for the dayside heightintegrated Pedersen and Hall conductances vertical profiles as displayed in Fig. 4.

From this one can derive the following scaling relations:

$$
\begin{aligned}
& \Sigma_{P} \propto M^{-1}, \\
& \Sigma_{H} \propto M^{-4 / 3} .
\end{aligned}
$$

They indicate that the conductances increase with decreasing magnetic field. The Cowling conductance, defined via

$\sigma_{C}=\sigma_{P}+\frac{\sigma_{H}^{2}}{\sigma_{P}}$

scales as

$\Sigma_{C} \propto M^{-5 / 3}$

The scaling relations derived here are in very good agreement with similar relations derived by Richmond (1995) in a different context.

On the nightside the ionospheric electron density $n_{e}(z)$ depends on the precipitation rate and energy of magnetospheric electrons and ions, as well as their interaction with the upper 


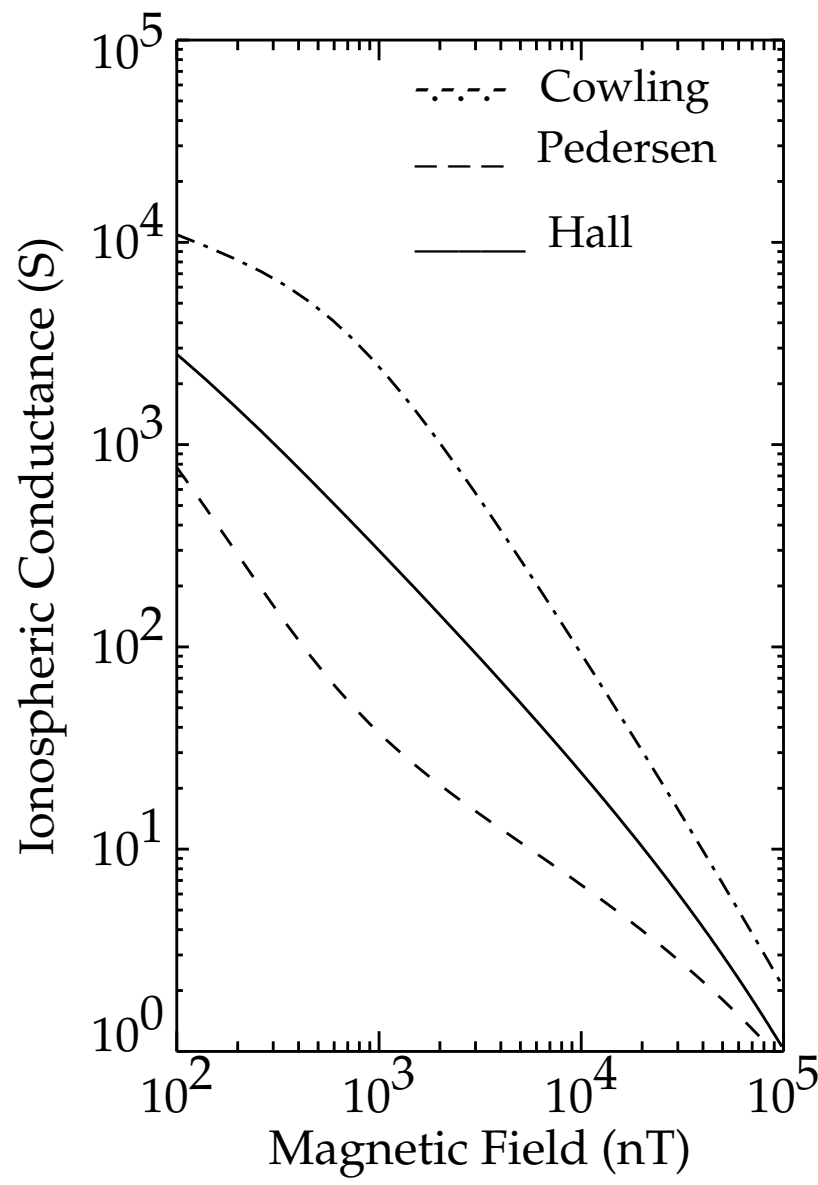

Fig. 4. Dependence of the dayside ionospheric conductances on the ionospheric magnetic field magnitude.

atmosphere. In order to derive a first simple scaling relation for these complex processes we note that the maximum ionospheric electron density $n_{\mathrm{e}, \max }$ scales with the precipitation rate, which certainly depends on the particles' loss cone $\lambda_{L}: n_{\mathrm{e}, \max } \propto \sin \lambda_{L}$ The width of the loss cone is independent from the particles' mass, charge, and energy (e.g. Baumjohann and Treumann, 1996). For a dipole field situation as assumed here the loss cone only depends on the field line radius via

$\sin ^{2} \lambda_{L}=\left(4 \cdot L^{6}-3 \cdot L^{5}\right)^{-1 / 2}$,

where $\mathrm{L}$ is the McIlwain parameter of the field line. The $\mathrm{L}$-value depends on the latitude $\lambda_{\text {Inter }}$ where the field line intersects the Earth's surface: $L=\cos ^{-2} \lambda_{\text {Inter }}$.

Discussing the polar electrojets we are interested in highlatitudes only where $\mathrm{L}$-values are about $\mathrm{L}=6$ and larger. For these polar situations Eq. (18) can be approximated as

$\sin ^{2} \lambda_{L}=\left(4 \cdot L^{6}\right)^{-1 / 2} \propto L^{-3}$.

Furthermore, assuming that the intersection latitude is identical with the polar cap latitude, that is $\lambda_{\text {Inter }}=\vartheta$, gives one a scaling relation

$\Sigma_{0} \propto n_{\mathrm{e}, \max } \propto \sin \lambda_{L} \propto M^{3 \gamma-3 / 2}$, where $\Sigma_{0}$ is the parameter introduced in Eq. (7).

Combining the relations (20), (7), (14), and (15) results into the following scalings for the nightside polar ionospheric conductances:

$\Sigma_{P} \propto M^{3 \gamma-5 / 2}$

$\Sigma_{H} \propto M^{3 \gamma-17 / 6}$.

There is no need to derive a corresponding relation for the Cowling conductivity as the equatorial electrojet is a dayside phenomenon.

Having derived scaling laws for the nightside ionospheric conductances, Eqs. (21) and (22), and the saturation ionospheric electric field, Eq. (7), allows one to deduce a scaling law for the polar electrojet magnetic field:

$b_{G} \propto M^{-\gamma+1 / 2}$

or, adopting the Siscoe and Chen (1975) case $\gamma=1 / 3$,

$b_{G} \propto M^{1 / 6}$,

that is the ground magnetic field of the polar electrojet increases weakly with increasing internal magnetic field.

The above scaling relations are based on several assumptions, such as the independence of $n_{e}(z)$ on $M$ for dayside conditions. Furthermore, only a first attempt has been made to estimate the magnetic field dependence of the influence of energetic particle precipitation on the nightside ionospheric conductivity. However, for the time being we take Eq. (23) as a suitable first scaling law for the external magnetic field variation due to the polar electrojets. But it should be kept in mind that this scaling relation has been derived based on assumptions with respect to magnetospheric structure, convection pattern, and particle precipitation.

With the above derived scaling law we can now discuss the time evolution of the external magnetic field contribution to the geomagnetic field. Again, using the dipole moments derived from the SINT800 record (Guyodo and Valet, 1999), assuming a present day polar electrojet strength of $200 \mathrm{nT}$, and an internal field at high latitudes of $50000 \mathrm{nT}$. Figure 5 displays the relative strength $\mathrm{r}_{\mathrm{PEJ}} \propto \mathrm{M}^{-5 / 6}$ of the polar electrojet field compared to the internal field contribution over the past 800000 years; here $\gamma=1 / 3$ has been assumed. Figure 5 indicates that the polar electrojet contribution was almost stable during the time interval represented by the SINT800 record. Only during the Matuyama-Brunhes transition some 780000 years ago did the relative strength of the PEJ significantly increase. Though the absolute PEJ strength was most probably smaller than today, its contribution to the total field was more pronounced, as the internal contribution decays faster with $\mathrm{M}$ than the external contribution. It would require a decrease of the internal contribution by about a factor of 300 during a polarity transition to reach an equal contribution of both the internal and external field. 
Table 1. Scaling relations for various magnetospheric parameters.

\begin{tabular}{lllll}
\hline & General & $\gamma=1 / 3$ & $\gamma=0$ & $\gamma=1 / 2$ \\
\hline$R_{M P}$ & $M^{1 / 3}$ & $M^{1 / 3}$ & $M^{1 / 3}$ & $M^{1 / 3}$ \\
$R_{P P}$ & $M^{3 \gamma-11 / 6}$ & $M^{-5 / 6}$ & $M^{-11 / 6}$ & $M^{-1 / 3}$ \\
$R_{T}$ & $M^{\gamma}$ & $M^{1 / 3}$ & & $M^{1 / 2}$ \\
$\cos \vartheta$ & $M^{\gamma-1 / 2}$ & $M^{-1 / 6}$ & $M^{-1 / 2}$ & \\
$D_{s t}$ & $M^{2 \gamma}$ & $M^{2 / 3}$ & & $M$ \\
$E_{I o n o, s}$ & $M^{\gamma+11 / 6} / \Sigma_{0}$ & $M^{13 / 6} / \Sigma_{0}$ & $M^{11 / 6} / \Sigma_{0}$ & $M^{7 / 3} / \Sigma_{0}$ \\
$b_{G}$ & $M^{-\gamma+1 / 2}$ & $M^{1 / 6}$ & $M^{1 / 2}$ & \\
$b_{E E J}$ & $M^{-2 / 3}$ & $M^{-2 / 3}$ & $M^{-2 / 3}$ & $M^{-2 / 3}$ \\
\hline
\end{tabular}

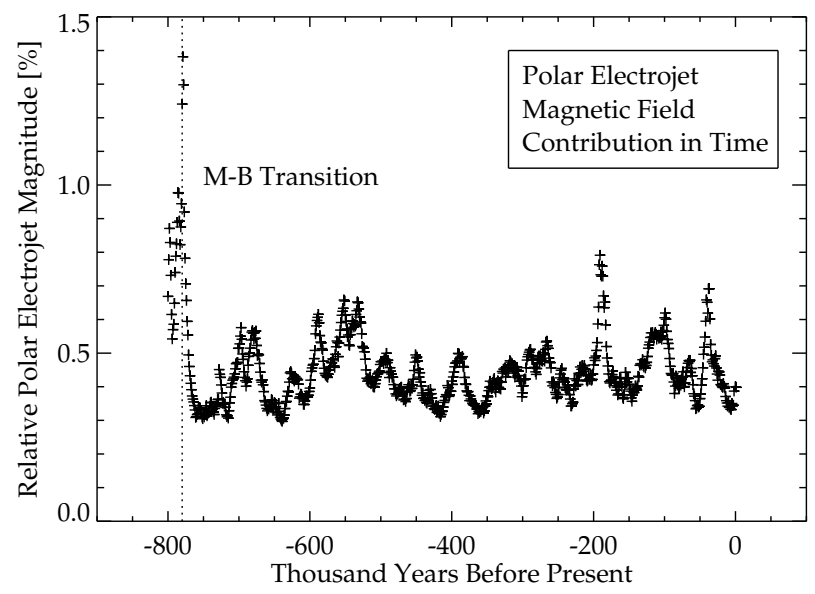

Fig. 5. Estimated relative polar electrojet strength over the past 800000 years.

\section{The equatorial electrojet}

Thermal tides (e.g. Volland, 1988), driven by temperature differences between the dayside and nightside atmosphere are the cause of the large-scale ionospheric Sq current system with ground-magnetic variations of the order of $10 \mathrm{nT}$. Near the geomagnetic equator a spatially confined current system develops, the equatorial electrojet (EEJ). Typical EEJ perturbation fields are of the order of $100 \mathrm{nT}$ (e.g. Untiedt, 1967; Hesse, 1982; Onwumechili, 1997). Its spatial localization to about $500 \mathrm{~km}$ around the magnetic equator results from space charge effects causing an effective conductivity, the Cowling conductivity $\sigma_{C}$, determining the strength of this current system. From Fig. 4 a scaling relation

$$
\Sigma_{C} \propto M^{-5 / 3}
$$

is appropriate for the Cowling conductance.

Thermal tides driving the EEJ are independent of the background magnetic field, but the electromotive force $\boldsymbol{u} \times \boldsymbol{B}$ driving the electric currents is proportional to M; here $\boldsymbol{u}$ is the tidal wind field. Thus, the EEJ strength scales as

$b_{E E J} \propto M^{-2 / 3}$.

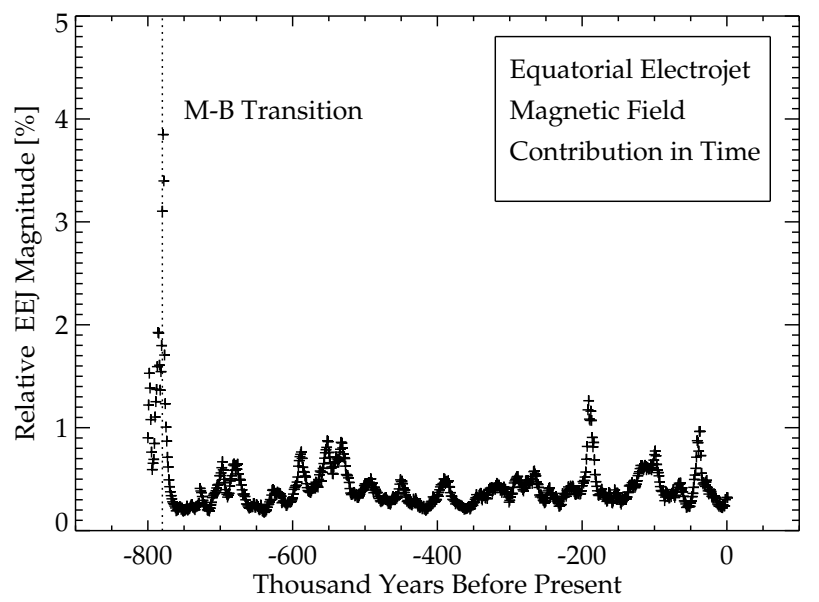

Fig. 6. Estimated relative equatorial electrojet strength over the past 800000 years.

We therefore conclude that the EEJ intensity increases significantly with decreasing magnetic field, that is during polarity transitions. The temporal evolution of this geomagnetic field contribution over the past 800000 years is displayed in Fig. 6, assuming a present day EEJ strength of $100 \mathrm{nT}$ and an internal field at the equator of $31000 \mathrm{nT}$. EEJ related ground magnetic fields of the order of $400-500 \mathrm{nT}$ are not unrealistic. The ratio external to internal field can be of the order of 1/20. The EEJ may become an important contributor to the total field during polarity transitions. It should be noted that the limitations of the scaling of the ionospheric conductances mentioned above do not apply for the EEJ case, as the dayside ionization of the upper atmosphere is mainly caused by solar UV and X-radiation.

\section{Summary and conclusions}

Scaling relations (see Table 1) for major magnetospheric parameters and ionospheric current systems and their dependence on the geomagnetic field have been critically assessed and new ones derived, assuming an interaction of the solar wind with a dipolar geomagnetic field. Their temporal 
evolution over the past 800000 years has been considered using estimates of the dipole strength of the Earth magnetic field based on relative paleointensity values of the SINT800 record compiled by Guyodo and Valet (1999). We conclude that, for example, the mean magnetopause distance did not change dramatically in the past. Current extreme positions of this major magnetospheric boundary are well within the range one expects under paleomagnetic field conditions.

We also derived a new scaling law for the plasmapause position, indicating that the relative size of the plasmasphere increases with decreasing moment. This is due to the fact that the corotation electric field decreases less rapidly with $\mathrm{M}$ than the saturation convection field. We conjecture that the paleomagnetosphere during a reversal is thus much more rotation dominated than at the present time and possibly exhibits features reminiscent to the Jovian magnetosphere. However, this conjecture needs further confirmation which is beyond the scope of this work.

Different from previous work we argue that the ring current magnetic field contribution decreases with decreasing geomagnetic field. This has implications for the interpretation of periods of extremely rapid field changes observed in paleomagnetic records during times of polarity transitions (Coe and Prevot, 1989; Coe et al., 1995), in that we cannot support the idea of ring current magnetic fields being responsible for these observed changes as advocated by, for example, Ultré-Guérard and Achache (1995) or Jackson (1995).

As a possible alternative we discuss polar and equatorial electrojets and derive proper scaling relations for their contribution to the external geomagnetic field. Based on a simple model we derive a scaling relation indicating that the polar electrojet fields exhibit a weak dependence from the internal dipole moment and decrease proportional to the decrease of the internal field. This scaling is mainly influenced by a saturation of the transpolar potential (e.g. Siscoe et al., 2002) governing the ionospheric electric field. Though the ionospheric conductances increase with decreasing dipole moment this saturation effect leads to the weak, positive dependence of the PEJ on M.

However, the relative contribution of the external field increases with decreasing internal field, which may be of importance especially during intervals of polarity transitions. At equatorial latitudes the EEJ is becoming much more pronounced, but never reaches a strength comparable to the internal field contribution.

From our considerations of the various sources of geomagnetic variations we conclude that the secular change of the dipole component of the geomagnetic field is of minor importance compared to the overall solar wind induced variability of the magnetosphere.

We would like to point out that our scaling relations are only first approaches to the problem. They are based on assuming a dipole magnetosphere, and convection electric fields similar in their topology, as in the present magnetosphere. Relaxing these limitations by modelling the magnetosphere-ionosphere system in more detail under con- ditions of a polarity transition is necessary to fully understand the problem.

Acknowledgements. KHG is grateful to George Siscoe for illuminating discussions of the problem addressed in this publication. This work has been conducted under the Priority Programme "Geomagnetic variations: Space-time structure, processes, and effects on system Earth" of the Deutsche Forschungsgemeinschaft. Financial support under contract GL 142-12/1 is gratefully acknowledged.

Topical Editor T. Pulkkinen thanks three referees for their help in evaluating this paper.

\section{References}

Allen, J., Sauer, H., Franck, L., and Reiff, P.: 14 November 1989: Effects of the March 1989 solar activity, EOS Trans. AGU, 14791486, 1989.

Baumjohann, W. and Treumann, R.: Basic Space Plasma Physics, 33f, Imperial College Press, London, 1996.

Brice, N. M.,: Bulk motion in the magnetosphere, J. Geophys. Res., 72, 5193-5201, 1967.

Boström, R.: A model of the auroral electrojet, J. Geophys. Res., 69, 4983-5000, 1964.

Burton, R. K., McPherron, R. L., and Russell, C. T.: An empirical relationship between interplanetary conditions and Dst, J. Geophys. Res., 80, 4204-4214, 1975.

Coe, R. S. and Prevot, M.: Evidence suggesting extremely rapid field variation during a geomagnetic reversal, Earth Planet. Sci. Lett., 92, 292-298, 1989.

Coe, R. S.: M. Prevot, and P Camps, New evidence for extraordinarily rapid change of the geomagnetic field during a reversal, Nature 374, 687-692, 1995.

Fukushima, N.: Generalized theorem of no ground magnetic effect of vertical current connected with Pedersen currents in the uniform conductivity ionosphere, Rep. Ionos. Space Res. Jpn., 30, 35-40, 1976.

Gazis, P. R.: Long-term enhancements in the solar wind speed, J. Geophys. Res., 101, 415-424, 1996.

Glassmeier, K. H.: Ground-based observations of field-aligned currents in the auroral zone: Methods and results, Ann. Geophys. 5, 115-126, 1987.

Glassmeier, K. H.: Currents in Mercury's Magnetosphere, Geophysical Monograph 118, 371-380, Am. Geophys. Union, Washington, 2000.

Guyodo, Y. and Valet, J. P.: Global changes in intensity of the Earths magnetic field during the past $800 \mathrm{kyr}$, Nature, 399, 249-252, 1999.

Hesse, D.: An investigation of the equatorial electrojet by means of ground-based magnetic measurements in Brazil, Ann. Geophys. 38, 315-320, 1982.

Hill, T. W., Dessler, A. J., and Wolf, R. A.: Mercury and Mars: The role of ionospheric conductivity in the acceleration of magnetospheric particles, Geophys. Res. Lett., 3, 429-432, 1976.

Jackson, A.: Storm in a lava flow? Nature, 377, 685-686, 1995.

Kamide, Y. and Baumjohann, W.: Magnetosphere-Ionosphere Coupling, Springer-Verlag, Berlin, 1993.

Kelley, M. C.: The Earth's Ionosphere, Academic Press, San Diego, 1989.

Laakso, H. and Jarva, M.: Position and Motion of the Plasmapause, J. Atm. Terr. Phys., 63, 1171-1178, 2001. 
Merrill, R. T. and McFadden, P. L.: Geomagnetic polarity transitions, Rev. Geophys. 37, 201-226, 1999.

Moldwin, M. B., Downward, L.,Rassoul, H. K., Amin, R., and Anderson, R. R.: A new model of the location of the plasmapause: CRRES results, J. Geophys. Res., 107, (A11), 1339, doi:10.1029/2001JA009211, 2002.

Onwumechili, C. A.: The equatorial electrojet, Gordon and Breach, Amsterdam, 1997.

Pirjola, R.: Fundamentals about the flow of geomagnetically induced currents in a power system applicable to estimating space weather risks and designing remedies, J. Atmos. Terr. Phys., 64, 1967-1972, 2002.

Richmond, A. D.: Ionospheric electrodynamics, in: Handbook of Atmospheric Electroidynamics, edited by: Volland, H., Vol. II, 260, CRC Press, Boca Raton, 1995.

Roelof, E. G. and Sibeck, D. G.: Magnetopause shape as a bivariate function of interplanetary magnetic field $B_{z}$ and solar wind dynamic pressure J. Geophys. Res. 98, 21 412, 1993.

Russell, C. T., Baker, D. N., and Slavin, J. A.: The magnetosphere of Mercury, edited by: Vilas, F., Chapman, C. P., and Matthwes, M. S., Mercury, Arizona University Press, Tuscon, 514561,1988

Russell, C. T., Le, G.,Chi, P., Zhou, X.-W., Shue, J.-H., Petrinec, S. M., Song, P., Fenrich, F. R., and Luhmann, J. G.: The extreme compression of the magnetosphere on 4 May 1998, as observed by the polar spacecraft, Adv. Space Res., 25, 1369-1375, 2000.

Sckopke, N.: A general relation between the energy of trapped particles and the disturbance field near the Earth, J. Geophys. Res. 71, 3225-3230, 1966.

Siebert, M.: Auswirkungen der säkularen Änderung des Erdmagnetischen Hauptfeldes auf Form und Lage der Magnetosphäre und die Stärke der erdmagnetischen Aktivität, Abhandlungen der Braunschweigischen Wissenschaftlichen Gesellschaft, 37, 281, 1977.
Siscoe, G. L. and Chen, C. K.: The paleomagnetosphere, J. Geophys. Res. 80, 4675-4680, 1975.

Siscoe, G. L., Erikson, G. M., Sonnerup, B. U.Ö., Maynard, N. C., Schoendorf, J. A., Siebert, K. D., Weimer, D. R., White, W. W., and Wilson, G. R.: Hill model of transpolar potential saturation: Comparisons with MHD simulations, J. Geophys. Res., 107(A6), SMP 8-1, doi:10.1029/2001JA000109, 2002.

Ultré-Guérard. P. and Achache, J.: Core flow instabilities and geomagnetic storms during reversals: The Steens mountain impulsive field variations revisited, Earth Planet. Sci. Lett. 135, 91-99, 1995.

Untiedt, J.: A model of the equatorial electrojet involving meridional currents, J. Geophys. Res. 72, 5799-5810, 1967.

Vasyliunas, V. M.: Mathematical models of magnetospheric convection and its coupling to the ionosphere, in: Particles and Fields in the Magnetosphere, edited by: McCormac, B. M., 6071, D. Reidel, Hinghamn, Mass., 1970.

Vogt, J. and Glassmeier, K. H.: On the location of trapped particle populations in quadrupole magnetospheres, J. Geophys. Res., 105, 13063-13071, 2000.

Vogt, J. and Glassmeier, K. H.: Modelling the paleomagnetosphere: Strategy and first results, Adv. Space Res. , 28, 863-868, 2001.

Volland, H.: Atmospheric Tidal and Planetary Waves, Kluver Academic Publisher, Dordrecht, 1988.

Wolf, R. A.: Magnetospheric configuration, in: Introduction to Space Physics, edited by: Kivelson, M. G. and Russell, C. T., Cambridge University Press, Cambridge, 1995. 